erst recht zu unmöglichen Vorstellungen. Schon 1934 hatte G a m o w ${ }^{11}$ auf der Londoner Tagung ausdrücklich darauf hingewiesen, daß der Gewinn an Zerfallsenergie, der die Emission eines ${ }^{12} \mathrm{C}$-Kernes an Stelle dreier in einer Zerfallsreihe aufeinanderfolgender $\alpha$-Teilchen begünstigte, mehr als wettgemacht würde durch die geringe Wahrscheinlichkeit, die dreimal größere Masse durch den dreimal höheren Potentialwall hindurchzubringen. Bei der Emission eines ${ }^{8} \mathrm{Be}$ statt zweier $\alpha$-Teilchen würde aber noch nicht einmal nennenswerte Bindungsenergie gewonnen, so daß eine solche Möglichkeit völlig entfiel.

Vielleicht daß solche Einwände, die von den Physikern des Instituts den Chemikern gegen die Entstehung von Radium-Isotopen gemacht wurden, dazu beigetragen haben, vielleicht daß auch Hahns übergroße Sorgfalt im Experimentieren allein dazu ausreichte - , jedenfalls überprüften Hahn und Straßmann ihre drei Ra-Isomere aufs neue, um ganz sicher zu erweisen, daß es sich dabei um Radium handelte. Denn daß auch dies Ergebnis, wenn es sich bewahrheitet hätte, für die physikalischen Vorstellungen vom Kernbau hochinteressant gewesen wäre, steht nach dem Vorangehenden außer Zweifel. Im Dezember 1938 äußerte Hahn dem Verf. gegenüber gesprächsweise zum ersten Male die Möglichkeit, daß die fraglichen Ra-Isomere sich eher wie Barium zu verhalten schienen. Eine sichere Aussage schien ihm noch nicht möglich. Zu einer ernsthaften Diskussion einer solchen Möglichkeit kam es noch nicht. Als wir Anfang Januar 1939 aus den Weihnachtsferien zurückkehrten, war bereits alles entschieden, die Ent-

11 G. G a m ow , Int. Conf. on Physics, London 1934.

12 O. H a hn u. F. St r a $§ \mathrm{~m}$ an $n$, Naturwiss. 27, 11 [1939], eingegangen am 22. Dez. 1938.

13 S. F l ü g g e u. G. v. D r o s t e, Z. physik. Chemie, Abt. B, 42, 274 [1939], eingegangen am 22. Jan. 1939 .

14 G. v. D r o s te, Naturwiss. 27, 198 [1939], eingegangen am 17. März 1939. stehung des Bariums gesichert und innerhalb weniger Tage in den ,Naturwissenschaften“ publiziert ${ }^{12}$. Und nun ging es wie mit dem Ei des Columbus: Innerhalb weniger Wochen war nicht nur von den Physikern im Hause überlegt, wie eine solche Spaltung möglich sei ${ }^{13}$, sondern auch ihr experimenteller Nachweis an Hand der Trümmer ${ }^{14}$ erbracht. In anderen Instituten ${ }^{15}$ ergab sich dasselbe Bild; mit klarem zeitlichen Vorsprung erreichten $\mathrm{Me}$ it $\mathrm{n}$ e r und $\mathrm{Fr}$ is $\mathrm{ch}^{\mathbf{1 3}}$ die unbestreitbare Priorität in Deutung und physikalischem Nachweis, während die ersten amerikanischen Arbeiten wenige Wochen danach die Spalten von Physical Review zu füllen begannen, und schließlich auch Paris mit einem entscheidend wichtigen neuen Motiv in den Chor einfiel, dem Nachweis der von Anbeginn vermuteten Spaltungsneutronen durch $\mathrm{J}$ o $\mathrm{l}$ i o t und seine. Mitarbeiter ${ }^{\mathbf{1 7}}$.

Man ist versucht, auf die mannigfachen Irrwege, welche der großen Entdeckung voraufgingen, das kürzlich von L. Rosenfeld angezogene Zitat zu gebrauchen: ,Pitié pour nous qui combattons toujours aux frontières de l'illimité et de l'avenir; pitié pour nos erreurs, pitié pour nos péchés" —, aber es war auch ein fruchtbarer Irrtum, aus dem eine viel intensivere Beschäftigung der Physiker mit der Frage der Isomerie entsprang, als es sonst vielleicht der Fall gewesen wäre, einer Frage, deren Verknüpfung mit dem Problem der Kernspaltung sich am Ende als Irrtum erwies.

15 Z. B. O. R. Fris ch, Nature [London] 143, 276 [1939], eingegangen im Januar 1939. R. D. F ow l e r u. R. W. D o d s o n, ebenda 143, 233 [1939]; Physic. Rev. 55, 417 [1939], eingegangen am 3. Febr. 1939.

W. Jentschke u. F. Prankl, Naturwiss. 27, 134 [1939], eingegangen am 14. Febr. 1939.

16 L. Meitner u. O. R. Frisch, Nature [London] 143, 239 [1939], eingegangen im Januar 1939, sowie $O$. R. F r i s c h ${ }^{\mathbf{1 5}}$.

17 M. Dod é, H. v. H a lban, F. Joliot u. L. K ow arski, C. R. hebd. Séances Acad. Sci. 208, 995 [1939].

\title{
Bemerkungen zum natürlichen Aufbau und zur Häufigkeitsverteilung der chemischen Elemente
}

\author{
Von Stefan Meyer \\ (Z. Naturforschg. 4 a, 84-87 [1949]; eingegangen am 5. Januar 1949)
}

Otto Hahn zum 70. Geburtstag zugeeignet

W Jenn man von der Genesis der chemischen Grundstoffe spricht, so denkt man gewöhnlich nur an den systematischen Aufbau aus den kleinsten Bausteinen, den Protonen und Neutronen, wie er vor und zum Teil vielleicht auch nach der Abtrennung der Erde von der Sonne unter ungeheueren Energietönungen vor sich gegangen sein mag ${ }^{1}$. Auf der Erde ken- nen wir gegenwärtig keinen natürlich verlaufenden Vorgang, der einen systematischen Aufbau

1 S. z. B. Zusammenstellungen bei S. F lü g g e u. J. Mattauch, Kernphysikalische Tabellen, SpringerVerlag, 1942: R. Sond e r, Z. Kristallogr.57,611 [1923]: Z. anorg. allg. Chem. 192, 257 [1930]; Gerlands Beitr. Geophysik 136, 319 [1932]; Schweiz. mineral. petrogr. Mitt. 28, 528 [1948]; H. E. S u e s s, Z. Naturforschg. $\mathbf{2 a}, 311,604$ [1947]. 
der Elemente aus Protonen und Neutronen schafft. Wohl aber haben die natürlichen radioaktiven Erscheinungen uns gelehrt, daß auf der Erde Artverwandlungen in irdischen Zeiten derart vor sich gehen, daß Abbauten das Maßgebliche und für die Häufigkeitsverteilung Ausschlaggebende sind.

Wir haben also von vornherein für die Gesetzmäßigkeiten in den Häufigkeitszahlen der Elemente zwei entgegengesetzte Momente zu unterscheiden *:

1. Systematischer Aufbau. Anstieg der Massenzahlen $(A)$ der artgleichen ${ }^{2}$ Elemente, schrittweise um $2 p+2 n$ und weiterhin ${ }^{3}$ immer vorherrschender um $2 p+4 n$.

Bei diesem Aufbau, der offenbar schon vor der Geburt der Erde stattfand, ist eine Bevorzugung der Stoffe mit geraden Massen- und Ordnungszahlen $(\mathrm{Gg})$ unverkennbar.

2. Ein systematischer Abbau, in Schritten, wie sie die radioaktiven Prozesse lehren: $\alpha, \alpha, \alpha, \ldots$ und $\alpha, \beta, \beta, \alpha$. Gesetzt, es gäbe in gleicher Art, wie wir es bei den radioaktiven Substanzen kennen, vom ${ }_{82}^{206} \mathrm{~Pb}$ und ${ }_{82}^{208} \mathrm{~Pb}$ abwärts noch einen entsprechenden weiteren Abbau, so zeigt die nachstehende Tab. 1 einen verblüffend einfachen analogen Gang.**

Für die Stoffe mit ungeradem $A$ ist ein solcher Abbau nicht durchwegs so klar ersichtlich.

3. Dazu kommt noch im Sinne der Entdeckung von O. Hahn und F. Straßmann die Aufspaltung der Atome mit den höchsten Ordnungszahlen $(Z)$ in Bruchstücke von der Art mittelschwerer Atome.

Da es zur Zeit der Erdentstehung auch seither ,ausgestorbene" Transurane gegeben haben mag, so können durch ihre stabilen Bruchstücke die Häufigkeiten einzelner Isotope der Jetztzeit beeinflußt sein. Inwieweit Aufspaltungen und somit Atombrocken solcher Herkunft bei der dermaligen irdischen Häufigkeitsverteilung zur Geltung kommen, ist bisher kaum einzuschätzen.

2 Artgleich bedeutet: Gg, Gu, Uu oder Ug. Gerade Massenzahl $=\mathrm{G}$, ungerade Massenzahl $=\mathrm{U} ; \mathrm{g}=$ gerade, $\mathrm{u}=$ ungerade Ordnungszahl.

3 Vgl. Mitt. Radiuminst. Nr. 456; S.-B. Akad. Wiss. Wien, Abt. II a 156, 624 [1947]; Acta physic. Austr. 2, 109 [1948].

* Die Häufigkeitsverteilung auf der Erde wurde bei Abtrennung des Mondes zuungunsten leichterer Elemente noch etwas modifiziert.
Daß bei Beachtung aller dieser Umstände Unregelmäßigkeiten in der Systematik der Häufigkeitsverteilungen durch gegenläufige Entstehungsweisen gedeutet werden können, ist zu erwarten und soll an einigen speziellen Beispielen erläutert werden.

Vor der Entdeckung der Isotopie waren die „Umstellungen“ im periodischen System mit den Atomgewichten als Basis:

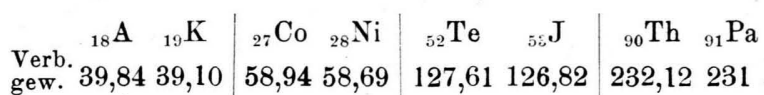

ein Gegenstand des Interesses. Nachher hat man sich schnell mit der Erklärung als befriedigt abgefunden, daß durch verschiedene Vermischung von Isotopen und durch die Packeffekte sowohl die Abweichungen von den Ganzzahligkeiten als auch die Umstellungen sich erklären lassen. Ganz so einfach liegen aber die Verhältnisse nicht. Übrigens sind $\mathrm{J}$, Th, Pa Reinelemente.

Beim radioaktiven Zerfall findet sich wiederholt das Schema: $\alpha, \beta, \beta, \alpha$, z. B.

$$
\mathrm{UI}-\alpha-\mathrm{UX}_{1}-\beta-\mathrm{UX}_{2}-\beta-\mathrm{UII}-a-\mathrm{Io}
$$

$\mathrm{Th}-\alpha-\mathrm{MsTh}_{1}-\beta-\mathrm{MsTh}_{2}-\beta-\mathrm{RdTh}-a-\mathrm{ThX}$.

In derartigen Fällen ist immer der zweite $\beta$ Strahler kürzerlebig, d. h. minder häufig als der vorhergehende ${ }^{4}$.

Der Fall: A rgon-Ka liu m

\begin{tabular}{|c|c|c|c|c|c|c|c|}
\hline Es ist: & & A & & & & $\mathrm{K}$ & \\
\hline $\begin{array}{l}\text { Ordnungszahl } \\
\text { Atomgewicht }\end{array}$ & & $\begin{array}{c}18 \\
39,914\end{array}$ & & & & $\begin{array}{l}19 \\
39,096\end{array}$ & \\
\hline Isotope & 36 & 38 & 40 & & 39 & 40 & 41 \\
\hline Häufigkeit $\%$ & 0,31 & 0,06 & 99,63 & & 93,4 & 0,01 & 6,6 \\
\hline Es ist: & & . & & & & & \\
\hline $\begin{array}{l}\text { Ordnungszahl } \\
\text { Atomgewicht }\end{array}$ & & & & & & & \\
\hline Isotope & 40 & 42 & 43 & 44 & 46 & 48 & \\
\hline Häufigkeit $\%$ & 99,96 & 0,64 & 0,15 & 2,07 & 0,003 & 0,1 & 19 . \\
\hline
\end{tabular}

Alle drei Elemente enthalten ein Isotop der Masse 40. Beim $\mathrm{K}$ ist es ein Gu-Kern. Von dieser Art sind außer ${ }_{1}^{2} \mathrm{He} \quad{ }_{3}^{8} \mathrm{Li} \quad{ }_{5}^{10} \mathrm{~B} \quad{ }_{7}^{14} \mathrm{~N}$ unter den natürlichen Kernen nur die $\beta$-strahlenden ${ }_{19}^{40} \mathrm{~K}{ }_{71}^{176} \mathrm{Cp}$ bekannt, und unter den natürlichen radioaktiven sind es nur die sehr kurzlebigen RaE, Th C", ThC, At, RaC', Fr, Ms Th, ${ }_{2}$, UZ.

4 S.-B. Akad. Wiss. Wien, Abt. II a 124, 249 [1915]; Elster-Geitel-Festschrift 1915, S. 146.

** Daß, ausgelöst durch kosmische Strahlen, aus verschiedenen Elementen auch $\alpha$-Strahlen auftreten könnten, wurde in Mitt. Radiuminst. Nr. 457; S.-B. Akad. Wiss. Wien, Abt. II a 156, 649 [1947] betont und seither durch H. E. H u n l e y, Nature [London] 161, 356 [1948], experimentell bestätigt. 
Th-Familie $(A=4 n)$

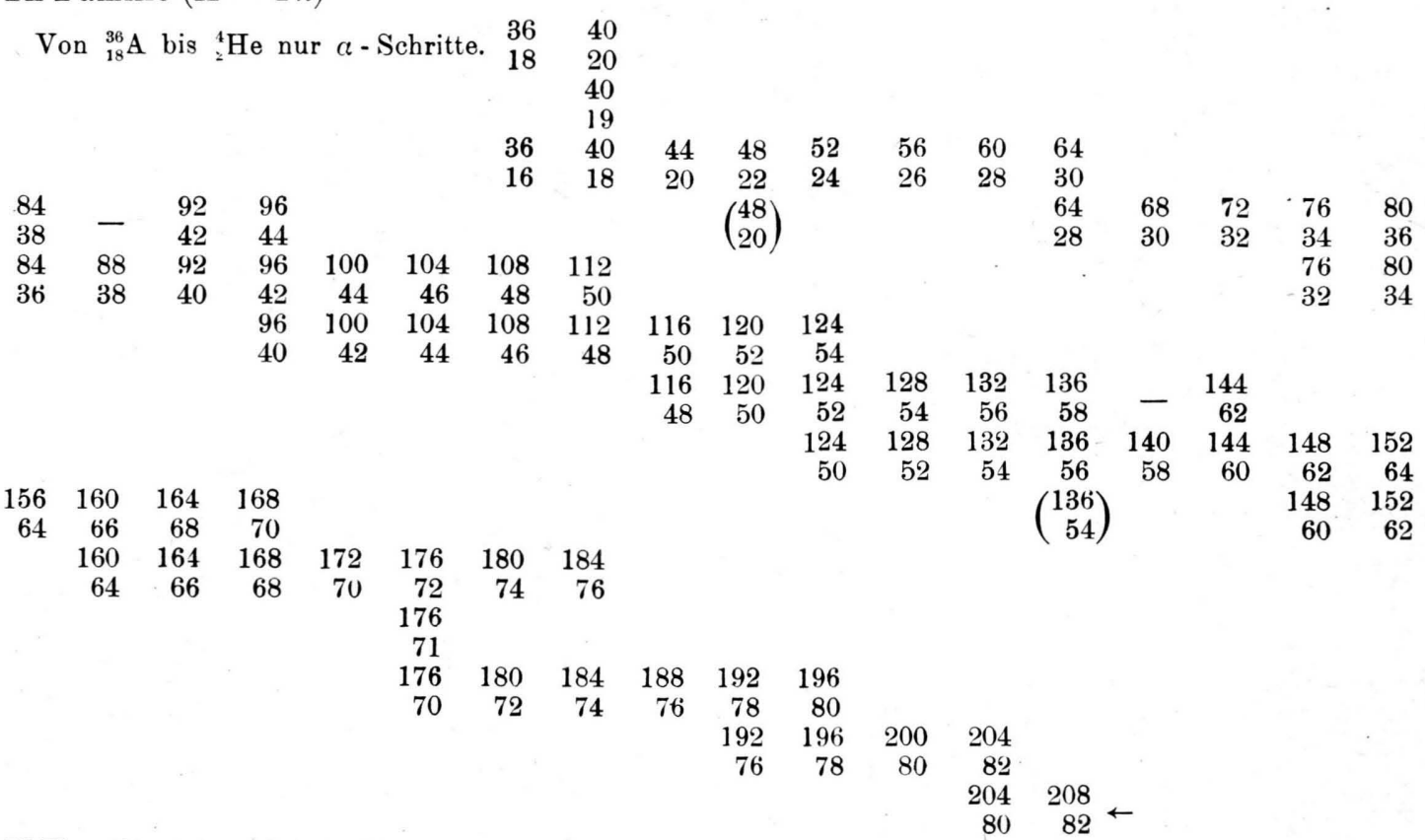

U-Familie $(A=4 n+2)$

Von ${ }_{22}^{46} \mathrm{Ti}$ bis ${ }_{8}^{18} \mathrm{O}$ nur $a$-Schritte. $\begin{array}{llll}46 & 50 & 54 & 58\end{array}$

$\begin{array}{lllllllll}22 & 24 & 26 & 28 & & & & & \\ 46 & 50 & \mathbf{5 4} & \mathbf{5 8} & 62 & 66 & \mathbf{7 0} & \mathbf{7 4} & \mathbf{7 8} \\ 20 & 22 & \mathbf{2 4} & 26 & 28 & 30 & 32 & \mathbf{3 4} & 36 \\ & & & & & & \mathbf{7 0} & \mathbf{7 4} & \mathbf{7 8} \\ & & & & & & 30 & 32 & 34\end{array}$

$\begin{array}{llllll}86 & 90 & 94 & 98 & 102 & 106\end{array}$

$\begin{array}{rrrrr}44 & 46 & 48 & & \\ 98 & 102 & 106 & 110 & 114\end{array}$

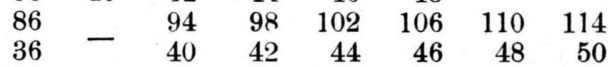

$\begin{array}{rrrrrr}110 & 114 & 118 & 122 & 126 & 130\end{array}$

$\begin{array}{rrrrrrrrrrrrrr}158 & 162 & & & & & & & & 50 & 52 & 54 & 5 \hbar & 5 \\ 66 & 68 & & & & & & & & & & 130 & 134 & 138 \\ 158 & 162 & 166 & 170 & 174 & & & & & & & 52 & 54 & \\ 64 & 66 & 68 & 70 & 72 & & & & & & & & \\ & & & 170 & 174 & 178 & 182 & 186 & & & & & \\ & & & 68 & 70 & 72 & 74 & 76 & & & & & \\ & & & & & & & 186 & 190 & 194 & 198 & & \\ & & & & & & & 74 & 76 & 78 & 80 & & \\ & & & & & & & & & & 198 & 202 & 206 \\ & & & & & & & & & & 78 & 80 & 82\end{array}$

Tab.1. Hypothetische Fortsetzung der Th- und U-Familie ${ }^{5}$. Die Kerne sind der Kürze halber nur durch Massen- und Kernladungszahl gekennzeichnet. ${ }_{20}^{48} \mathrm{Ca}$ und ${ }_{54}^{136} \mathrm{X}$ fallen heraus.

Man könnte daher in Analogie zu den radioaktiven Schemen $\alpha, \beta, \beta, \alpha$ ansetzen

$$
{ }_{20}^{44} \mathrm{Ca}-a-{ }_{18}^{40} \mathrm{~A}-\beta-{ }_{19}^{40} \mathrm{~K}-\beta-{ }_{20}^{40} \mathrm{Ca}-a-,
$$

wie dies seit vielen Jahren geschah.

Wie dort ist der zweite $\beta$-Strahler der kürzerlebige und daher minder häufige. Rückkehr in die

5 Mitt. Radiuminst. Nr. 457; S.-B. Akad. Wiss. Wien, Abt. II a 156, 641 [1947]; Acta physic. Austr. 2, 124 [1948]. gleiche Plejade von ${ }^{44} \mathrm{Ca}$ in ${ }^{40} \mathrm{Ca}$ liefert hier ein stabileres Produkt. Die geringe Häufigkeit von ersterem läßt eine gewisse Instabilität erwarten. Daß bisher keine $\beta$-Strahlung aus ${ }^{40} \mathrm{~A}$ und keine $\alpha$-Strahlung aus ${ }^{44} \mathrm{Ca}$ bekannt sind, besagt nicht allzu viel. Es kann auch ,strahlenlose“ Umwandlungen geben, d. h. solche, aus denen mit unseren Beobachtungsmitteln keine entsprechenden Wirkungen bisher festgestellt wurden, oder gar 
Veränderungen um $2 p+2 n$, ohne daß es zu einer He-Kern-Bildung kommt.

Sieht man aber in obiger Zusammenstellung von den Kernen der Massenzahl $40 \mathrm{ab}$, d. h. betrachtet man diese als anderer Herkunft, so verschwindet die Umstellung bei $\mathrm{A}-\mathrm{K}$ und auch der übermäßige Sprung vom Ca zum Sc sowie der von $\mathrm{Cl} \mathrm{zu} \mathrm{A}$.

Die Genesis der verschiedenen K-Isotope kann sonach verschiedener und gegenläufiger Art sein, und das vermöchte vielleicht auch die Beobachtungen von E. Gledits $\mathrm{ch}^{6}$ über den Elektronen-Einfang in den Kalium-Kern und die daraus resultierenden Erscheinungen verständlicher zu machen.

Wie an anderer Stelle gesagt wurde ${ }^{3}$, lassen sich ähnliche Deutungen auch für die erwähnten anderen „Umstellungen“ geben.

Näher eingegangen sei noch auf den Fall des Cassiopeium, Rubidium und Samarium.

\section{Cassiopeiu m}

Neben ${ }^{175} \mathrm{Cp}$ mit $97,5 \%$ Häufigkeit gibt es das viel weniger stabile und daher weit seltenere ${ }_{71}^{176} \mathrm{Cp}$. So wie ${ }_{19}^{40} \mathrm{~K}$ und einige kurzlebige radioaktive Kerne der großen radioaktiven Reihen, ist es ein $\beta$-Strahler der Art Gu.

Die Zusammenhänge würden sich zwanglos wie beim $\mathrm{K}$ nach dem Schema

$$
{ }_{72}^{180} \mathrm{Hf}-a-{ }_{70}^{176} \mathrm{Yb}-\beta-{ }_{71}^{178} \mathrm{Cp}-\beta-{ }_{72}^{176} \mathrm{Hf}
$$

deuten lassen.

$$
\text { Rubidium }
$$

$\mathrm{Rb}$ ist neben $\mathrm{K}$ ein schon sehr früh als $\beta$-strahlend erkanntes Element. Man unterscheidet derzeit bloß die zwei natürlichen Isotope: ${ }_{37}^{85} \mathrm{Rb}$ mit $72,8 \%$ und ${ }_{57}^{87} \mathrm{Rb}$ mit $27,2 \%$.

Man weist derzeit die natürliche $\beta$-Strahlung dem Isotop $87 \mathrm{zu}$, und dies hat eine besondere Stütze in der Arbeit von O. Hahn, F. Stra B mann und Walling ${ }^{7}$ gefunden, die nebeneinander ${ }_{37}^{87} \mathrm{Rb}$ und ${ }_{38}^{87} \mathrm{Sr}$ im gleichen Material mit Hilfe von M atta u chs Massenspektrogrammen feststellen konnten.

Nun ist das einigermaßen auffallend für einen $\beta$-Strahler, der andererseits so stabil ist, daß ${ }^{87} \mathrm{Rb}$

6 E. G l e dit s c h, Physic. Rev. 72, 640 [1947]; vgl. auch J. H. J. Ṕ o o l e, Nature [London] 162, 775 [1948].

7 O. Hahn, F. Straßmann u. Walling, Naturwiss. 25, 189 [1937]; ebenda J. M a t t a u c h. eine Häufigkeit der gleichen Größenordnung hat wie ${ }^{85} \mathrm{Rb}$. Die anderen derartigen natürlichen radioaktiven Stoffe, $\mathrm{K}$ und $\mathrm{Cp}$, gehören der Art $\mathrm{Gu}$ an; man hätte daher für das $\beta$-strahlende $\mathrm{Rb}$ eher ein seltenes ${ }^{86} \mathrm{Rb}$ erwarten können. Die $\mathrm{Zu}$ ordnung zu ${ }^{87} \mathrm{Rb}$ war nur von Mattauch nach seiner Isobarenregel vorausgesehen worden.

Vielleicht bietet andererseits das ${ }^{187} \mathrm{Re}$, das als $\beta$-strahlend gemeldet wurde ${ }^{8}$, einen Parallelfall. Dabei mag es beachtenswert sein, daß ${ }^{87} \mathrm{Rb}$ und ${ }^{187} \mathrm{Re}$ der sogenannten $4 n+3$-Reihe angehören, die Isotope mit $A=85$ und $A=185 \operatorname{der} 4 n+1$-Reihe.

\section{S a m a ri u m}

Als Isotope sind nach $\mathrm{Matta} \mathrm{ch}^{9}$ bekannt:

$\begin{array}{llllllll}A \ldots \ldots 14 & 147 & 148 & 149 & 150 & 152 & 154\end{array}$

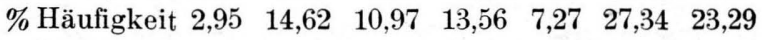

Beim Nd finden sich dazu als Isobare 144, 148, 150. Man könnte demnach wiederum an das Schema denken:

$$
{ }_{62}^{148} \mathrm{Sm}-a-{ }_{60}^{144} \mathrm{Nd}-\beta-{ }_{61}^{144} \mathrm{Pm}-\beta-{ }_{62}^{144} \mathrm{Sm},
$$

wovon Nd bereits mehrmals als $\beta$-strahlend signalisiert wurde und $Z=61$ (Illinium = Promethium) bestimmt sehr selten und kurzlebig ist.

Man hat bis vor kurzem die $\alpha$-Strahlung dem Isotop ${ }^{148} \mathrm{Sm}$ zugeordnet. Neuerdings wird die $\alpha$ Aktivität dem ${ }^{152} \mathrm{Sm}$ zugeschrieben ${ }^{10}$. Ein analoges Schema wie obiges für ${ }^{148} \mathrm{Sm}$ läßt sich ebensogut für ${ }^{152} \mathrm{Sm}$ mit der Verwandlung in ${ }^{148} \mathrm{Nd}$ aufstellen, und das träfe auch für ${ }^{154} \mathrm{Sm}$ und ${ }^{150} \mathrm{Nd}$ zu. Dabei wären in obigem Sinne 152 und 148 der Th-Familie, dagegen 154 der U-Familie zuzurechnen.

In allen Fällen würde die Rückkehr in die gleiche Plejade zu einem Stoff geringerer Stabilität (Häufigkeit) führen.

Es wäre auch denkbar, daß die Sm- $\alpha$-Aktivität nicht einheitlich ist, und zwar nach der Herkunft aus 154, 152, 148 verschieden. Die Massen 150 und 144 kommen wegen des Fehlens von Sm-Isotopen der Massen 146 und 140 nicht in Frage.

Es mag nicht bedeutungslos sein, daß gerade die Häufigkeiten der Gg-Isotope des $\mathrm{Nd}^{-}$(ebenso wie die des Ca, das in der Diskussion über $\mathrm{K}$ herangezogen ist) Unregelmäßigkeiten zeigen.

${ }^{8}$ L i b b y, Physic. Rev. 73, 487 [1948]; A. H. W. A t e n jr., Nature [London] 161, 1019 [1948].

9 J. M a t t a u c h, Z. Naturforschg. 3a, 105 [1948].

10 A. J. D e m p s t e r, Physic. Rev. 73, 1125 [1948]; N. F e a t h e r, Nature [London] 161, 412 [1948]. 